\title{
How to identify a hyperelastic constitutive equation for rubber-like materials with multiaxial tension-torsion experiments
}

\author{
A.-S. Lectez ${ }^{\mathrm{a}, \mathrm{b}}$, E. Verron ${ }^{\mathrm{a}, *}, \mathrm{~B}$. Huneau ${ }^{\mathrm{a}}$ \\ a Institut de Recherche en Génie Civil et Mécanique (GeM), UMR CNRS 6183, Ecole Centrale de Nantes, BP 92101, 44321 Nantes cedex 3, France \\ ${ }^{\mathrm{b}}$ Direction Engineering Systèmes et Gestion de l'Energie Renault, 1 avenue du Golf, 78288 Guyancourt, France
}

\begin{abstract}
This paper discusses the different approaches that can be used to determine the strain energy density of a given rubber-like material based on tension-torsion experimental results. More precisely, the aim is to answer the question: how to handle the measured macroscopic quantities, i.e. load and torque, to determine the constitutive equation with the less possible assumptions? The method initially proposed by Penn and Kearsley [Trans. Soc. Rheol. 20 (1976) 227-238] is adopted: the strain energy derivatives with respect to kinematical quantities have to be calculated in terms of the measured load and torque. Here, we propose to consider different sets of kinematical quantities to overcome the incoherence encountered with the classical Cauchy-Green strain invariants $I_{1}$ and $I_{2}$. Two new sets are considered: the principal stretch ratios and two specific invariants of the logarithmic (true) Hencky strain tensor. The corresponding derivations coupled with new experimental results permit (i) to calculate the Cauchy stress tensor on the outer surface of the cylindrical samples, and (ii) to demonstrate that a wellconditioned set of kinematical quantities must be adopted to determine the strain energy density. It is proved here that the principal stretch ratios are good candidates to express and determine the strain energy density with tension-torsion experiments.
\end{abstract}

\author{
Keywords: \\ Rubber-like materials \\ Hyperelasticity \\ Tension-torsion experiments \\ Experimental stresses \\ Strain energy function
}

\section{Introduction}

In service, industrial elastomeric parts are subjected to severe multiaxial mechanical loading conditions, thus the use of finite element calculations during their design necessitates robust and accurate hyperelastic constitutive equations. The easiest and most common method adopted to determine the most relevant constitutive equation for a given material consists in performing uniaxial tension-compression experiments. Nevertheless, identifying the corresponding material parameters with such experimental data leads to poor prediction of the mechanical responses for non-uniaxial loading conditions (see for example $[20,30]$ ). The first method to overcome this limitation is to enrich the experimental database with supplementary data issued from other loading condition experiments, such as pure shear, simple shear or equibiaxial tension. The major drawback is that both specific specimens and experimental set-ups are needed. The second method consists in performing experiments that combine different simple loading conditions; in this case, unique geometry of specimen and experimental set-up are sufficient to obtain several

\footnotetext{
* Corresponding author. Tel.: +33240376842.

E-mail address: erwan.verron@ec-nantes.fr (E. Verron).
}

deformation states. In this context, biaxial experiments such as bulge tests $[19,28,31,35]$ or square sheets tensile tests $[3,15,27,33]$ are revealed difficult to perform due to both the complexity of experimental set-ups and the inhomogeneity of the deformation in the specimens. On the contrary, simultaneous tension-torsion experiments are a relevant alternative to consider combined loading conditions: the cylindrical geometry of the specimen is simple and commercial testing machines are nowadays available.

Considering such experiments, the problem reduces to the determination of the constitutive equation, i.e. the stress-strain relationship, from the measurement of load and torque for prescribed extension and angle of twist. As the kinematics of cylinders subjected to simultaneous tension and torsion is well-established, the derivation of strain from extension and angle of twist is obvious. The difficulty of the method consists in the derivation of stress from measured force and torque. Practically three approaches can be adopted to identify the constitutive equation:

(i) choosing a strain energy function and identifying the corresponding parameters by comparison between experimental and theoretical force and torque data,

(ii) determining the stress state from experimental data, choosing a relevant strain energy function and identifying the corresponding parameters with the stress-strain experimental data, 
(iii) determining the strain energy derivatives from experimental data, choosing a relevant strain energy function and identifying the corresponding parameters with the strain energy derivatives-strain data.

The approach (i) is classical and is not developed in this paper; its main drawback is that the strain energy function must be presupposed without any clue. The two other approaches do not require to presuppose the strain energy function.

In the case of homogeneous deformation, like uniaxial tension, pure shear, equibiaxial tension or biaxial tension, approach (ii) is often used since the relation between stress and load is straightforward (see for example $[32,16]$ ). In this context, a specific type of plot is widely considered, presenting reduced stress versus extension and referred to as the Mooney plot ([34,10,4] for example). Nevertheless, determining experimental stresses for tension-torsion tests is not obvious because of the inhomogeneity of deformation in the radial direction of cylindrical specimens. Penn and Kearsley [26] derived the method to determine experimental derivatives of the strain energy with respect to strain invariants at the outer radius of the cylinder for the special case of pure torsion (length is held constant). Later, Humphrey et al. [13] applied the method to pre-extension followed by torsion of a transversely isotropic cylinder. Knowing the experimental strain energy derivatives, it becomes easy to calculate stresses. However, to the authors' knowledge, no stress-strain curve has been published for large strain combined tension-torsion experiments on rubber-like materials. Thus, the first objective of this paper is to present experimental stresses data at the outer radius of an elastomeric cylinder subjected to pre-extension followed by torsion.

Approach (iii) appears to be more complicated. Firstly, a choice has to be made concerning chosen quantities with respect to which the strain energy derivatives are calculated. Most authors consider uniaxial and biaxial tension experiments, and choose the two first invariants of Cauchy-Green strain tensors $I_{1}$ and $I_{2}$ $[1,5,8,14,15,27,23,34,17]$. Secondly, depending on the chosen quantities and the considered loading conditions, strong hypotheses have to be made. For example, it is possible to calculate a combination of strain energy derivatives with respect to $I_{1}$ and $I_{2}$ in the case of uniaxial tension, but each one is undetermined without further assumptions. Rivlin and Saunders [27] supposed that $\partial W / \partial I_{1}$ is constant while $\partial W / \partial I_{2}$ is a function of $I_{2}$ only. Thirdly, a rigorous analysis of experimental strain energy derivatives is not facilitated by the coupling between invariants, and by the scattering of experimental results, particularly for small strain $[27,9,6]$. Concerning the heterogeneous case of tension-torsion tests, some authors ([21] for example) applied the method presented by Penn and Kearsley [26] to build experimental strain energy derivatives curves. These data are restricted to pure torsion (uniaxial extension maintained to 1 ), and are always expressed in terms of $I_{1}$ and $I_{2}$. Thus, the second objective of this paper is to calculate strain energy derivatives with respect to various mechanical quantities (invariants of tensors, stretch ratios) for various extensions followed by torsion and to discuss the best choice for the determination of the most relevant constitutive equation.

In the next section, all the ingredients of the method are presented: kinematics and governing equations of a cylinder under quasi-static tension-torsion loading conditions, the derivatives of the strain energy function (similarly than in [26]), and finally the derivation of stress. Attention is drawn on the possibility to choose different sets of strain invariants to obtain the derivatives of the strain energy. Considering new tension-torsion experimental data, results obtained with the different sets of strain energy derivatives as well as results for stress are derived in Section 3 for different values of pre-extension followed by torsion. Finally, Section 4 proposes a discussion on the advantages and drawbacks of each approach; in particular, experimental data issued from strain energy derivatives confirm the importance of choosing a well-conditioned set of invariants, as emphasized by Criscione [6].

\section{Relationships between local quantities and experimental measurement of load and torque}

\subsection{Governing equations of a cylinder under quasi-static isochoric uniaxial tension and torsion}

These equations are well-established since Rivlin and Saunders [27], and are briefly recalled here for clarity.

Fig. 1 presents the notations for the tension-torsion of a homogeneous cylinder in both undeformed and deformed configurations.

Rubber-like materials being classically considered incompressible, the deformation is supposed isochoric. A homogeneous cylinder of initial length and radius $L$ and $A$ is considered. In the reference configuration $\left(C_{0}\right)$ a material point $M$ of the undeformed cylinder is positioned by its coordinates $(R, \Theta, Z)$ in the cylindrical coordinate system $\left(\boldsymbol{e}_{R}, \boldsymbol{e}_{\Theta}, \boldsymbol{e}_{Z}\right)$. The deformation consists in an axial displacement $u$ in the direction $\boldsymbol{e}_{Z}$ and an angle of twist $\phi$ around the same vector, applied on the top surface $S_{\text {sup }}$ while the bottom surface $S_{\text {inf }}$ is maintained and the lateral surface $S_{\text {lat }}$ remains free to move. The length and radius of the deformed cylinder are denoted $l$ and $a$ respectively. The point $M$ moves to $M^{\prime}$, and its coordinates are $(r, \theta, z)$ in the deformed cylindrical coordinate system $\left(\boldsymbol{e}_{r}, \boldsymbol{e}_{\theta}, \boldsymbol{e}_{z}\right)$. The displacement from $M$ to $M^{\prime}$ is given by

$r=\frac{R}{\sqrt{\lambda}}, \quad \theta=\Theta+\tau Z, \quad z=\lambda Z$,

where $\lambda$ is the extension and $\tau$ is the angle per unit of undeformed length. They are uniform and defined as

$\lambda=\frac{l}{L}, \quad \tau=\frac{\phi}{L}$.

Consequently, the deformation gradient $\boldsymbol{F}$ is

$\boldsymbol{F}=\frac{1}{\sqrt{\lambda}}\left(\boldsymbol{e}_{r} \otimes \boldsymbol{e}_{R}+\boldsymbol{e}_{\theta} \otimes \boldsymbol{e}_{\Theta}\right)+\tau r \boldsymbol{e}_{\theta} \otimes \boldsymbol{e}_{Z}+\lambda \boldsymbol{e}_{Z} \otimes \boldsymbol{e}_{Z}$,

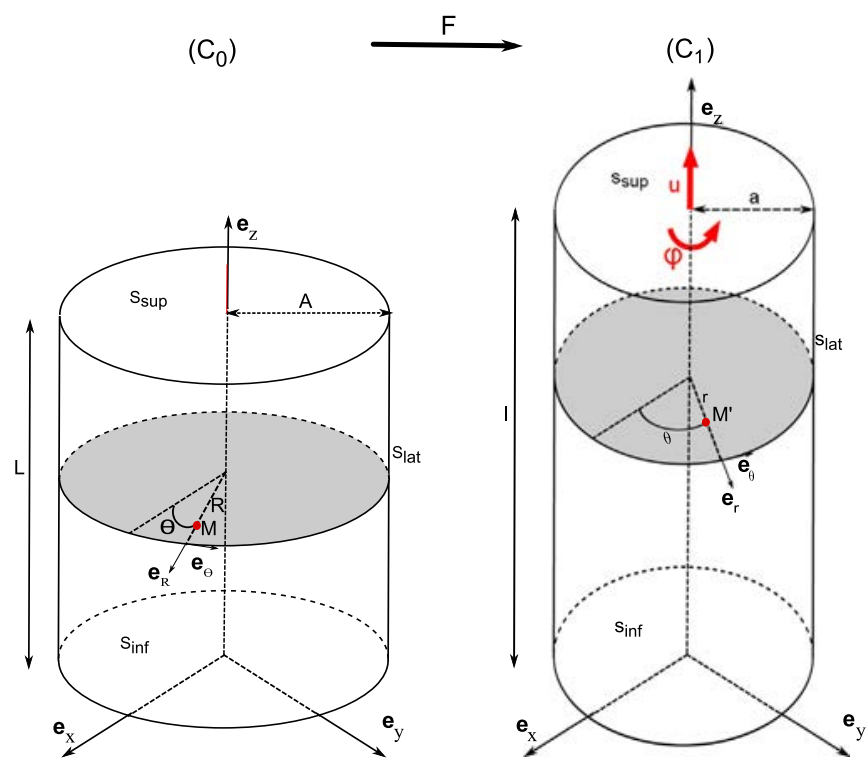

Fig. 1. Kinematics of a cylinder under uniaxial tension and torsion: notations. 
and the left Cauchy-Green strain tensor $\boldsymbol{B}$ has the following form:

$$
\begin{aligned}
\boldsymbol{B} & =\frac{1}{\lambda} \boldsymbol{e}_{r} \otimes \boldsymbol{e}_{r}+\left(\frac{1}{\lambda}+\tau^{2} r^{2}\right) \boldsymbol{e}_{\theta} \\
& \otimes \boldsymbol{e}_{\theta}+\lambda \tau r\left(\boldsymbol{e}_{\theta} \otimes \boldsymbol{e}_{z}+\boldsymbol{e}_{z} \otimes \boldsymbol{e}_{\theta}\right)+\lambda^{2} \boldsymbol{e}_{z} \otimes \boldsymbol{e}_{z} .
\end{aligned}
$$

Eq. (4) shows that the strain is entirely determined by the extension $\lambda$ and the twist angle per unit length $\tau$.

Next, we focus on the stress state: our aim is to determine the Cauchy stress tensor $\boldsymbol{\sigma}$. From the experimental point of view, the quantities measured are the load force $N$ and the torque $M$; they are respectively related to the Cauchy stress tensor components by

$N \boldsymbol{e}_{z}=\int_{S_{\text {sup }}} \boldsymbol{\sigma} \cdot \boldsymbol{e}_{z} d S=\int_{S_{\text {sup }}} \sigma_{z z} d S \boldsymbol{e}_{z}$

and

$M \boldsymbol{e}_{z}=\int_{S_{\text {sup }}} r \boldsymbol{e}_{r} \times\left(\boldsymbol{\sigma} \cdot \boldsymbol{e}_{\theta}\right) d S=\int_{S_{\text {sup }}} r \sigma_{\theta z} d S \boldsymbol{e}_{z}$.

Since $\boldsymbol{\sigma}$ is locally defined, it is not possible to bypass the integration and to express $\sigma_{\theta z}$ and $\sigma_{z z}$ as explicit functions of $N$ and $M$. Furthermore, without additional assumptions, there is no equations that involve the other stress components. We now restrict the investigation to the special case of homogeneous, incompressible, isotropic hyperelastic materials. Indeed, the mechanical response of the material is defined by strain energy function $W$ which, for isotropic materials, depends on the two first invariants of $\boldsymbol{B}$, denoted $I_{1}$ and $I_{2}$, and given by

$I_{1}=\tau^{2} r^{2}+\frac{2}{\lambda}+\lambda^{2}$

$I_{2}=\frac{\tau^{2} r^{2}}{\lambda}+\frac{1}{\lambda^{2}}+2 \lambda$

The Cauchy stress tensor derives from this strain energy function (see for example [12]). Denoting $W_{1}$ and $W_{2}$ the derivatives of $W$ with respect to $I_{1}$ and $I_{2}$ respectively,

$\boldsymbol{\sigma}=-p \mathbf{I}+2 W_{1} \boldsymbol{B}-2 W_{2} \boldsymbol{B}^{-\mathbf{1}}$

where $\boldsymbol{I}$ is the $3 \times 3$ identity tensor and $p$ is the Lagrange multiplier associated with the incompressibility constraint (often referred to as the hydrostatic pressure). For the tension-torsion strain tensor $\boldsymbol{B}$ given by Eq. (4), it leads to

$\sigma_{r r}(r)=2 W_{1} \lambda^{-1}-2 W_{2} \lambda-p$

$\sigma_{\theta \theta}(r)=2 W_{1}\left(\lambda^{-1}+\tau^{2} r^{2}\right)-2 W_{2} \lambda-p$

$\sigma_{z z}(r)=2 W_{1} \lambda^{2}-2 W_{2}\left(\lambda^{-2}+\frac{\tau^{2} r^{2}}{\lambda}\right)-p$

$\sigma_{\theta z}(r)=2 W_{1} \lambda \tau r+2 W_{2} \tau r$

$\sigma_{r \theta}(r)=0$

$\sigma_{r z}(r)=0$

In order to determine $p$, we consider the equilibrium equation in the radial direction

$\frac{d \sigma_{r r}(s)}{d s}+\frac{1}{s}\left(\sigma_{r r}(s)-\sigma_{\theta \theta}(s)\right)=0, \quad 0<s<a$

and the boundary condition on the lateral surface

$\sigma_{r r}(a)=0$

Integration of Eq. (16) between the radius $s=r$ and the lateral surface $s=a$ with the constitutive equations (10)-(11) and the boundary condition equation (17) permits to obtain the value of $p$ at $r$

$p(r)=2 \tau^{2} \int_{r}^{a} W_{1}(s) s d s+2 W_{1}(r) \lambda^{-1}-2 W_{2}(r) \lambda$,

and the diagonal components of $\sigma$

$$
\begin{aligned}
\sigma_{r r}(r) & =-2 \tau^{2} \int_{r}^{a} W_{1}(s) s d s \\
\sigma_{\theta \theta}(r) & =-2 \tau^{2} \int_{r}^{a} W_{1}(s) s d s+2 W_{1}(r) \tau^{2} r^{2} \\
\sigma_{z z}(r) & =2 W_{1}(r)\left(\lambda^{2}-\lambda^{-1}\right)-2 W_{2}(r)\left(\lambda^{-2}-\lambda+\frac{\tau^{2} r^{2}}{\lambda}\right) \\
& -2 \tau^{2} \int_{r}^{a} W_{1}(s) s d s .
\end{aligned}
$$

Then, it is possible to express $N$ in terms of the derivatives of the strain energy function

$$
\begin{aligned}
N= & 4 \pi\left(\lambda-\lambda^{-2}\right) \int_{0}^{a}\left(\lambda W_{1}(r)+W_{2}(r)\right) r d r \\
& -4 \pi \frac{\tau^{2}}{\lambda} \int_{0}^{a} W_{2}(r) r^{3} d r-4 \pi \tau^{2} \int_{0}^{a}\left[r \int_{r}^{a} W_{1}(s) s d s\right] d r,
\end{aligned}
$$

or, after integration by parts of the last term on the right-hand side,

$$
\begin{aligned}
N= & 4 \pi\left(\lambda-\lambda^{-2}\right) \int_{0}^{a} r\left(\lambda W_{1}(r)+W_{2}(r)\right) d r \\
& -2 \pi \frac{\tau^{2}}{\lambda} \int_{0}^{a}\left(2 W_{2}(r)+\lambda W_{1}(r)\right) r^{3} d r .
\end{aligned}
$$

Similarly, invoking Eqs. (6) $)_{2}$ and (13), the torque is

$M=4 \pi \tau \int_{0}^{a}\left(\lambda W_{1}(r)+W_{2}(r)\right) r^{3} d r$.

\subsection{Derivatives of the strain energy function}

Once the experiments performed, the quantities $\lambda, \tau, M$ and $N$ are known; in displacement driven experiments, the two former quantities are prescribed and the two latter ones are measured. As shown in Eqs. (10)-(15), the determination of the stress tensor necessitates the calculation of the strain energy derivatives $W_{1}$ and $W_{2}$. Nevertheless, these derivatives are functions of the radial position $r$ and their forms are not known. Thus, considering Eqs. (23)-(24) they can be expressed in terms of $N$ and $M$ only if further assumptions are adopted.

In the next paragraphs, the derivation of $W_{1}$ and $W_{2}$ and the corresponding assumptions are first presented, then the derivatives of $W$ with respect to the principal stretch ratios are established, and finally a new set of relevant strain invariants is introduced to propose a new approach of the problem.

\subsubsection{Derivatives of the strain energy with respect to $\left(I_{i}\right)_{i=1,2}$}

This derivation has been first introduced by Penn and Kearsley [26]. As shown by Eqs. (7)-(8), $I_{1}$ and $I_{2}$ are functions of $\lambda$ and $\tau r$; thus $W, W_{1}$ and $W_{2}$ are also functions of these two quantities. Performing the change of variables $u=\tau r$, Eqs. (23)-(24) become

$$
\begin{aligned}
N \tau^{2}= & 4 \pi\left(\lambda-\lambda^{-2}\right) \int_{0}^{\tau a}\left(\lambda W_{1}(\lambda, u)+W_{2}(\lambda, u)\right) u d u \\
& -\frac{2 \pi}{\lambda} \int_{0}^{\tau a}\left(2 W_{2}(\lambda, u)+\lambda W_{1}(\lambda, u)\right) u^{3} d u
\end{aligned}
$$


and

$M \tau^{3}=4 \pi \int_{0}^{\tau a}\left(\lambda W_{1}(\lambda, u)+W_{2}(\lambda, u)\right) u^{3} d u$.

In both equations, the right-hand side terms depend on $\tau$ only through the upper bound of the integrals.

The next step of the derivation consists in differentiating Eqs. (25)-(26) with respect to $\tau$. Nevertheless, it is possible only if an additional assumption is adopted: $\lambda$ must be set constant, i.e. only torsion around a constant pre-stretching must be considered. Then, applying the Leibniz integral rule, $W_{1}$ and $W_{2}$ can be evaluated at the outer undeformed radius $A$

$\left.W_{1}(\lambda, \tau A)=\frac{\lambda}{\tau^{2} A^{2}} T_{N}+2 \frac{1}{\lambda \tau^{2} A^{2}}+\frac{1}{\lambda}-\frac{\lambda^{2}}{\tau^{2} A^{2}}\right) T_{M}$

$\left.W_{2}(\lambda, \tau A)=-\frac{\lambda^{2}}{\tau^{2} A^{2}} T_{N}+\frac{2 \lambda^{3}}{\tau^{2} A^{2}}-\frac{2}{\tau^{2} A^{2}}-1\right) T_{M}$

where

$T_{N}=\frac{\lambda}{2 \pi \tau A^{2}} \frac{d\left(N \tau^{2}\right)}{d \tau}$ and $T_{M}=\frac{\lambda^{2}}{4 \pi \tau^{3} A^{4}} \frac{d\left(M \tau^{3}\right)}{d \tau}$.

Thus, the functions $N \tau^{2}$ and $M \tau^{3}$ can be measured experimentally, then differentiated with respect to $\tau$, and finally using Eq. (29) in Eqs. (27)-(28) leads to the derivatives of the strain energy with respect to $I_{1}$ and $I_{2}$.

\subsubsection{Derivatives of the strain energy with respect to the principal} stretch ratios $\left(\lambda_{i}\right)_{i=1,2,3}$

Some classical hyperelastic models are expressed in terms of the principal stretch ratios $\left(\lambda_{i}\right)_{i=1,2,3}$, for example the Ogden model [24]. In this case the strain energy function is explicitly written in terms of the principal stretch ratios, i.e. $W\left(\lambda_{1}, \lambda_{2}, \lambda_{3}\right)$ and the principal Cauchy stresses differences are

$\sigma_{i}-\sigma_{j}=\lambda_{i} \frac{\partial W}{\partial \lambda_{i}}-\lambda_{j} \frac{\partial W}{\partial \lambda_{j}} \quad(i, j)=(1,2,3)^{2}, \quad i \neq j$.

In the special case of incompressible materials, stretch ratios are related by $\lambda_{1} \lambda_{2} \lambda_{3}=1$, the strain energy function can be written as $\bar{W}\left(\lambda_{1}, \lambda_{3}\right)=W\left(\lambda_{1}, 1 /\left(\lambda_{1} \lambda_{3}\right), \lambda_{3}\right)$. Then, the principal Cauchy stresses differences become (see [25])

$\sigma_{1}-\sigma_{2}=\lambda_{1} \frac{\partial \bar{W}}{\partial \lambda_{1}}$

$\sigma_{3}-\sigma_{2}=\lambda_{3} \frac{\partial \bar{W}}{\partial \lambda_{3}}$

$\sigma_{3}-\sigma_{1}=\lambda_{3} \frac{\partial \bar{W}}{\partial \lambda_{3}}-\lambda_{1} \frac{\partial \bar{W}}{\partial \lambda_{1}}$

Moreover, recalling that the invariants are

$I_{1}=\lambda_{1}^{2}+\lambda_{2}^{2}+\lambda_{3}^{2}=\lambda_{1}^{2}+\lambda_{3}^{2}+\frac{1}{\lambda_{1}^{2} \lambda_{3}^{2}}$

$I_{2}=\lambda_{1}^{2} \lambda_{2}^{2}+\lambda_{2}^{2} \lambda_{3}^{2}+\lambda_{3}^{2} \lambda_{1}^{2}=\frac{1}{\lambda_{1}^{2}}+\frac{1}{\lambda_{3}^{2}}+\lambda_{1}^{2} \lambda_{3}^{2}$,

the derivatives of $\bar{W}$ with respect to $\lambda_{1}$ and $\lambda_{3}$ are related to $W_{1}$ and $W_{2}$ by

$\left.\left.\frac{\partial \bar{W}}{\partial \lambda_{1}}=2 \quad \lambda_{1}-\frac{1}{\lambda_{1}^{3} \lambda_{3}^{2}}\right) W_{1}+2 \quad \lambda_{1} \lambda_{3}^{2}-\frac{1}{\lambda_{1}^{3}}\right) W_{2}$

$\left.\left.\frac{\partial \bar{W}}{\partial \lambda_{3}}=2 \quad \lambda_{3}-\frac{1}{\lambda_{3}^{3} \lambda_{1}^{2}}\right) W_{1}+2 \quad \lambda_{1}^{2} \lambda_{3}-\frac{1}{\lambda_{3}^{3}}\right) W_{2}$
In the special case of simultaneous tension-torsion, the principal stretch ratios at the outer radius $A$ can be written as functions of $\lambda$ and $\tau$

$\lambda_{1}=\left[\frac{1}{2}\left(\lambda^{2}+\frac{1+\tau^{2} A^{2}}{\lambda}-\sqrt{\left(\lambda^{2}+\frac{1+\tau^{2} A^{2}}{\lambda}\right)^{2}-4 \lambda}\right)\right]^{1 / 2}$

$\lambda_{2}=\frac{1}{\sqrt{\lambda}}$

$\lambda_{3}=\left[\frac{1}{2}\left(\lambda^{2}+\frac{1+\tau^{2} A^{2}}{\lambda}+\sqrt{\left(\lambda^{2}+\frac{1+\tau^{2} A^{2}}{\lambda}\right)^{2}-4 \lambda}\right)\right]^{1 / 2}$.

Moreover, as the radial stress $\sigma_{2}$ is null on the outer radius, Eqs. (31)-(32) give the two non-zero principal Cauchy stresses at the outer radius

$\sigma_{1}=\lambda_{1} \frac{\partial \bar{W}}{\partial \lambda_{1}} \quad$ and $\quad \sigma_{3}=\lambda_{3} \frac{\partial \bar{W}}{\partial \lambda_{3}}$,

where the partial derivatives of $\bar{W}$ are given by Eqs. (36)-(37), and the principal stretch ratios $\lambda_{1}$ and $\lambda_{3}$ by Eqs. (38)-(40).

\subsubsection{Derivatives of the strain energy with respect to the invariants} $\left(K_{i}\right)_{i=2,3}$ of the Hencky strain tensor

Following the work of Criscione et al. [7], we propose here to use the Hencky strain tensor, also referred to as the true strain tensor or the logarithmic strain tensor, to analyze the tensiontorsion experimental results. Indeed, Criscione et al. [7] developed this theory to overcome the difficulties observed when classical hyperelastic models expressed in terms of $I_{1}$ and $I_{2}$ are considered to fit experimental data, due to the correlation of the quantities involved in the fitting process. The mathematical relevance of this framework has been recently investigated by Sendova and Walton [29].

The Hencky strain tensor noted $\boldsymbol{H}$ in the following is defined as

$\boldsymbol{H}=\ln \left(\boldsymbol{B}^{1 / 2}\right)$,

or also

$\boldsymbol{H}=\ln \boldsymbol{V}$

where $\boldsymbol{V}$ is the pure strain tensor issued from the left polar decomposition of the deformation gradient $\boldsymbol{F}=\boldsymbol{V} \boldsymbol{R}, \boldsymbol{R}$ being a rotation tensor. The invariants of $\boldsymbol{H}$ introduced by Criscione et al. [7] are

$K_{1}=\operatorname{tr} \boldsymbol{H}$

$K_{2}=\sqrt{\operatorname{dev} \boldsymbol{H}: \operatorname{dev} \boldsymbol{H}}$

$K_{3}=\frac{3 \sqrt{6}}{K_{2}^{3}} \operatorname{det}(\operatorname{dev} \boldsymbol{H})$,

where $\operatorname{dev} \bullet=\bullet-(\operatorname{tr} \bullet / 3) \boldsymbol{I}$ stands for the deviatoric operator. It is worth noting that each of these invariants admits a simple mechanical meaning: $K_{1}$ quantifies the amount of volumetric dilatation, $K_{2}$ quantifies the amount of distortion and $K_{3}$ describes the mode of distortion $\left(K_{3}=-1\right.$ for uniaxial compression and equibiaxial tension, $K_{3}=0$ for pure shear and $K_{3}=1$ for uniaxial tension). For incompressible materials, $K_{1}=1$, and then the strain energy only depends on $K_{2}$ and $K_{3}$. Note that in this case $\boldsymbol{H}$ is purely deviatoric, i.e. $\operatorname{dev} \boldsymbol{H}=\boldsymbol{H}$. In this case, $\left(K_{i}\right)_{i=2,3}$ admit simple expressions in terms of principal stretch ratios

$K_{2}=\sqrt{\left(\ln \lambda_{1}\right)^{2}+\left(\ln \lambda_{2}\right)^{2}+\left(\ln \lambda_{3}\right)^{2}}$ 
$K_{3}=\frac{3 \sqrt{6}}{K_{2}^{3}} \ln \lambda_{1} \ln \lambda_{2} \ln \lambda_{3}$.

Consider now a strain energy function written in terms of the Hencky strain invariants $W\left(K_{2}, K_{3}\right)$. It is to note that in the undeformed configuration, $K_{2}=0$, and then $K_{3}$ is undetermined; thus we set $W\left(0, K_{3}\right)=0$. The Cauchy stress is given by Criscione et al. [7]

$\boldsymbol{\sigma}=-p \boldsymbol{I}+\frac{1}{K_{2}} \frac{\partial W}{\partial K_{2}} \boldsymbol{H}+\frac{1}{K_{2}} \frac{\partial W}{\partial K_{3}} \boldsymbol{Y}$

where the second-order tensor $\boldsymbol{Y}$ stands for

$\boldsymbol{Y}=\frac{3 \sqrt{6}}{K_{2}^{2}} \boldsymbol{H}^{2}-3 \frac{K_{3}}{K_{2}} \boldsymbol{H}-\sqrt{6} \boldsymbol{I}$.

As emphasized by Criscione et al. [7], the terms of the Cauchy stress tensor in Eq. (49) are mutually orthogonal, i.e. $\boldsymbol{I}: \boldsymbol{H}, \boldsymbol{I}: \boldsymbol{Y}$ and $\boldsymbol{H}: \boldsymbol{Y}$ are null. Then, the strain energy derivatives with respect to $K_{2}$ and $K_{3}$ are given respectively by

$\frac{\partial W}{\partial K_{2}}=\frac{1}{K_{2}} \boldsymbol{\sigma}: \boldsymbol{H} \quad\left(=\frac{\sigma_{1} \ln \lambda_{1}+\sigma_{3} \ln \lambda_{3}}{K_{2}}\right)$,

and

$\frac{\partial W}{\partial K_{3}}=\frac{K_{2}}{9\left(1-K_{3}^{2}\right)} \boldsymbol{\sigma}: \boldsymbol{Y}$.

Remark 1. When $K_{3}^{2}$ tends to one, $\boldsymbol{Y}$ tends to zero. Without further assumptions, it is not possible to determine if $\partial W / \partial K_{3}$ is finite or not when $K_{3}^{2}=1$. However, recalling that $\sigma$ is finite and considering Eq. (49), $\partial W / \partial K_{3} \boldsymbol{Y}$ tends to zero. The experimental results for the quantity $\partial W / \partial K_{3}$ in the neighborhood of $K_{3}^{2}=1$ will be investigated in the Results section.

Finally, invoking Eq. (41) the strain energy derivatives in Eqs. (51)-(52) can be written in terms of the derivatives of $W$ with respect to the stretch ratios

$\left.\frac{\partial W}{\partial K_{2}}=\frac{1}{K_{2}} \lambda_{1} \ln \lambda_{1} \frac{\partial \bar{W}}{\partial \lambda_{1}}+\lambda_{3} \ln \lambda_{3} \frac{\partial \bar{W}}{\partial \lambda_{3}}\right)$,

and

$$
\begin{aligned}
\frac{\partial W}{\partial K_{3}}= & \frac{1}{9\left(1-K_{3}^{2}\right)}\left[\frac{3 \sqrt{6}}{K_{2}}\left(\ln \lambda_{1}\right)^{2}-\sqrt{6} K_{2}-3 K_{3} \ln \lambda_{1}\right) \lambda_{1} \frac{\partial \bar{W}}{\partial \lambda_{1}} \\
& \left.+\left(\frac{3 \sqrt{6}}{K_{2}}\left(\ln \lambda_{3}\right)^{2}-\sqrt{6} K_{2}-3 K_{3} \ln \lambda_{3}\right) \lambda_{3} \frac{\partial \bar{W}}{\partial \lambda_{3}}\right] .
\end{aligned}
$$

\subsubsection{Summary}

In the previous sections, we established the equations that give the derivatives of the strain energy function with respect to several mechanical quantities at the outer radius of a uniaxially extended cylinder subjected to torsion using only the global experimental results, i.e. the stretch $\lambda$, the angle of twist $\tau$, the force $N$ and the torque $M$. More precisely, these calculations necessitate the differentiation of $N$ and $M$ with respect to $\tau$. Moreover, once the derivatives calculated, it is possible to determine the components of the Cauchy stress tensor $\boldsymbol{\sigma}$.

Table 1 summarizes all the previous results, emphasizing the necessary equations for each quantity.

\section{Results}

The previous derivation is now applied to some experimental data. Two different types of experiments are considered: pure torsion, i.e. $\lambda=1$, and uniaxial pre-stretching, i.e. $\lambda>1$, followed
Table 1

Necessary equations to calculate strain energy derivatives and Cauchy stress components for tension-torsion experiments at the outer radius.

\begin{tabular}{ll}
\hline Quantity & Equations \\
\hline$\partial W / \partial I_{1}$ & $(27),(29)$ \\
$\partial W / \partial I_{2}$ & $(28),(29)$ \\
$\partial \bar{W} / \partial \lambda_{1}$ & $(36),(38),(40),(27)-(29)$ \\
$\partial \bar{W} / \partial \lambda_{3}$ & $(37),(38),(40),(27)-(29)$ \\
$\partial W / \partial K_{2}$ & $(53),(47),(36)-(37),(38)-(40),(27)-(29)$ \\
$\partial W / \partial K_{3}$ & $(54),(47)-(48),(36)-(37),(38)-(40),(27)-(29)$ \\
$\sigma_{\theta \theta}$ & $(20)$ for $r=a,(27)-(29)$ \\
$\sigma_{z z}$ & $(21)$ for $r=a,(27)-(29)$ \\
$\sigma_{\theta z}$ & $(13)$ for $r=a,(27)-(29)$ \\
\hline
\end{tabular}
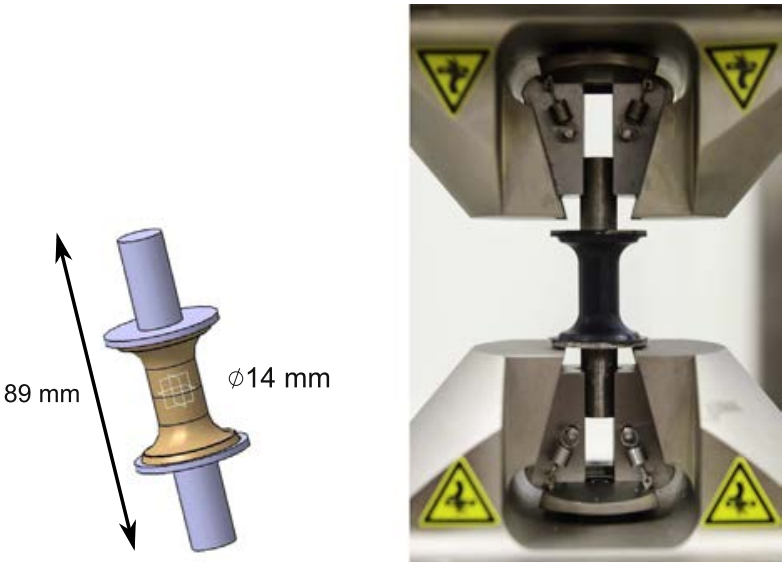

Fig. 2. Specimen for tension-torsion tests with its steel mounting fixtures, 3D view (left) and picture of the specimen in the tension-torsion machine (right).

by pure torsion. First, our experimental procedure is briefly described; then the equations summarized in Table 1 are applied to experimental data. The stress-strain curves at the outer radius are established to demonstrate the capability of our approach. Finally, the derivatives of the strain energy functions with respect to the different sets of invariants considered above are investigated.

\subsection{Experimental procedure}

As shown in Fig. 2, the specimens are dumbbells with a substantial cylindrical center zone. These samples have been especially designed for characterization under tension-torsion tests as explained in detail in Lectez et al. [18]. Particularly, they fulfill two mandatory requirements: the ability to reach large strain and to match the analytical solution of the infinite cylinder. Other convenient constraints have been considered in order to be used in our tension-torsion machine, the Instron Electropuls E10000; they are not detailed in the present paper.

The material studied here is a carbon black-filled Natural Rubber (NR)- Styrene Butadiene Rubber (SBR) blend. Before each test, Mullins effect is suppressed: the specimen is accommodated during five simultaneous tension-torsion loading/unloading cycles. The maximal imposed values are $\lambda_{\max }=2.5$ and $\tau_{\max }=$ $0.09 \mathrm{rad} \mathrm{mm}^{-1}$, where $\lambda_{\max }$ and $\tau_{\max }$ are respectively the maximum axial stretch ratio and the maximum angle per unit of length.

The strain rate during each test is set to $10^{-3} \mathrm{~s}^{-1}$, which can be considered as quasi-static loading conditions. Three kinds of tests are performed: pure torsion tests (the extension $\lambda$ is maintained equal to 1 ), pre-stretching followed by torsion, and simultaneous 

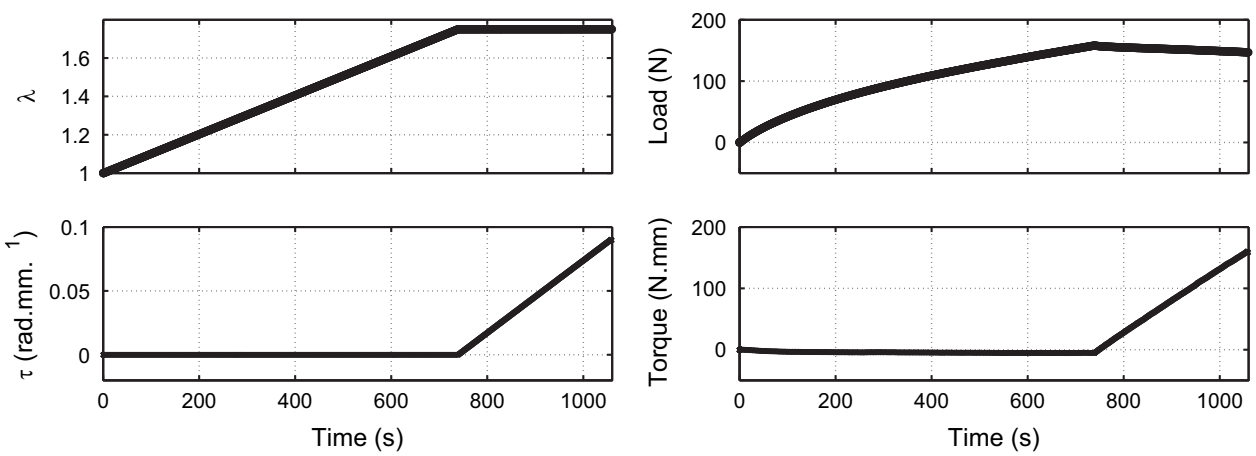

Fig. 3. Prescribed stretch ratio $\lambda$ and angle of twist per unit of length $\tau$ versus time (left). Measured load and torque (right).

uniaxial tension-torsion tests. Extension and angle of twist are prescribed and the corresponding torque and load are measured. Data are filtrated in order to reduce noise; each experiment is repeated three times and only the loading part of the cycles is considered. As an example, Fig. 3 presents the measured load and torque for an experiment that consists in pre-stretching $\lambda_{\max }=1.75$ followed by torsion $\tau_{\max }=0.09 \mathrm{rad} \mathrm{mm}^{-1}$.

\subsection{Cauchy stress tensor}

Using the methodology described in Section 2, it is first possible to obtain the stress-strain curves at the outer radius of the sample. As stated by Eqs. (13)-(15) and (19)-(21), the only non-zero components of the Cauchy stress tensor are the components in the tangential plane to the surface $\left(\boldsymbol{e}_{\theta}, \boldsymbol{e}_{z}\right)$, i.e. $\sigma_{\theta \theta}, \sigma_{\theta z}$ and $\sigma_{z z}$. Here, two cases are analyzed: pure torsion, then pre-stretching followed by torsion.

Pure torsion: For pure torsion, there is no uniaxial extension $\lambda=1$, and only the angle of twist is prescribed. The corresponding stress-strain curves are presented in Fig. 4. More precisely, it depicts the non-zero Cauchy (true) stress components as functions of the corresponding Hencky (true) strain components, i.e. $H_{\theta \theta}$, $H_{\theta z}$ and $H_{z z}$. It is revealed that for the studied strain range, each Cauchy stress component varies linearly with the corresponding Hencky strain. Moreover, we can also note that the axial stress component $\sigma_{z z}$ decreases as $H_{z z}$ increases and is negative; it reflects the well-know Poynting effect (see for example [2]).

Pre-stretching followed by torsion: This second type of loading conditions consists in prescribing a uniaxial pre-stretching followed by torsion. Two pre-stretching values are considered: $\lambda=1.75$ and 2.5 . Fig. 5 shows the evolution of the non-zero components of the Cauchy stress tensor with the angle of twist per unit of undeformed length $\tau$; $\tau$ is preferred to the components of $\boldsymbol{H}$ for more clarity in the drawings. First, it is verified that the initial value of the axial stress $\sigma_{z z}$ is equal to the axial stress measured for the pure uniaxial experiment at the same stretch level. Then, $\sigma_{z z}$ decreases quickly before reaching an almost constant value: this decrease in stress is induced by the relaxation of the axial stress $\sigma_{z z}$ due to viscous effects. Moreover, as $\sigma_{z z}$ reaches an almost constant level, it means that the contribution of torsion loading conditions on the axial stress is negligible as compared to the contribution of the pre-stretching; it is also observable by comparing the values of $\sigma_{z z}$ in Fig. 5 with the ones attained in pure torsion shown in Fig. 4. Moreover $\sigma_{\theta \theta}$ admits the same evolution as $\sigma_{z z}$, beginning from zero (uniaxial tension) and decreasing quickly before reaching a constant value. As for $\sigma_{\theta z}$, it takes positive values and varies linearly with $\tau$. The slope increases with the pre-extension.

\subsection{Derivatives of the strain energy function}

In the next sections, the derivatives of the strain energy functions with respect to the three mechanical quantities are calculated using equations given in Table 1.

\subsubsection{With respect to the classical Cauchy-Green strain invariants} $\left(I_{i}\right)_{i=1,2}$

Fig. 6 presents the evolution of $W_{1}$ and $W_{2}$ with respect to the corresponding invariants. More precisely, Fig. 6(a) and (b) corresponds to pure torsion and the abscissas are $I_{1}-3$ and $I_{2}-3$, and Fig. 6(c) and (d) corresponds to pre-stretching followed by torsion and the abscissas are the difference between the current values of the invariants and their values at the end of the prestretching, i.e. $I_{1}-\left(\lambda^{2}+2 \lambda^{-1}\right)$ and $I_{2}-\left(2 \lambda+\lambda^{-2}\right)$.

Pure torsion: In this case, $I_{1}=I_{2}$. As shown in Fig. 6(a) and (b) respectively, $W_{1}$ decreases very quickly to a constant positive value while $W_{2}$ increases very quickly to another constant positive value. The decrease in $W_{1}$ and the increase in $W_{2}$ are almost symmetrical about the horizontal axis. Moreover, the final values of $W_{1}$ and $W_{2}$ are consistent with classical values of MooneyRivlin parameters for such materials.

Similar results have been observed for example by Penn and Kearsley [26], and Fukahori and Seki [8]: $W_{1}$ and $W_{2}$ seem to tend to infinity as $I_{1}$ and $I_{2}$ tend to 3 , i.e. the undeformed configuration. To the authors' knowledge, only one published model has been proposed to predict such behavior: Obata et al. [23] defined $W_{1}$ and $W_{2}$ as functions of negative powers of $\left(I_{1}-3\right)$ and $\left(I_{2}-3\right)$. Nevertheless, such approach has never been further investigated.

Pre-stretching followed by torsion: The evolution of $W_{1}$ and $W_{2}$ during torsion after pre-stretching is respectively shown in Fig. 6(c) and (d) for two values of pre-stretching $\lambda=1.75$ and $\lambda=2.5$. Similarly than for pure torsion, $W_{1}$ and $W_{2}$ seem to tend to infinity for the lowest values of invariants, i.e. the values of the invariants at the end of pre-stretching. The most noticeable difference with pure torsion results is the change of sign of both derivatives of the strain energy functions.

Finally, considering the infinite limits of the four curves in Fig. 6 and recalling that no hyperelastic constitutive equations are able to reproduce such trends of $\left(\partial W / \partial I_{i}\right)_{i=1,2}$, the results raise questions on the relevance of the Cauchy-Green invariants in the present context.

\subsubsection{With respect to the stretch ratios $\left(\lambda_{i}\right)_{i=1,3}$}

Fig. 7 presents the evolution of the strain energy functions with respect to the stretch ratios, i.e. $\bar{W}_{1}=\partial \bar{W} / \partial \lambda_{1}$ and $\bar{W}_{3}=\partial \bar{W} / \partial \lambda_{3}$, for both pure torsion and pre-stretching followed by torsion.

Pure torsion: Fig. 7(a) and (b) shows the evolution of the strain energy derivatives with respect to stretch ratios in the case of pure torsion. $\bar{W}_{1}$ is always negative and decreases linearly with $\lambda_{1}$, while $\bar{W}_{3}$ is positive and increases as $\lambda_{3}$ increases. Contrary to $W_{1}$ and $W_{2}$, no singularities are observed.

Pre-stretching followed by torsion: After pre-stretching, as the deformation state is uniaxial the only non-zero stress is $\sigma_{z z}$, and 

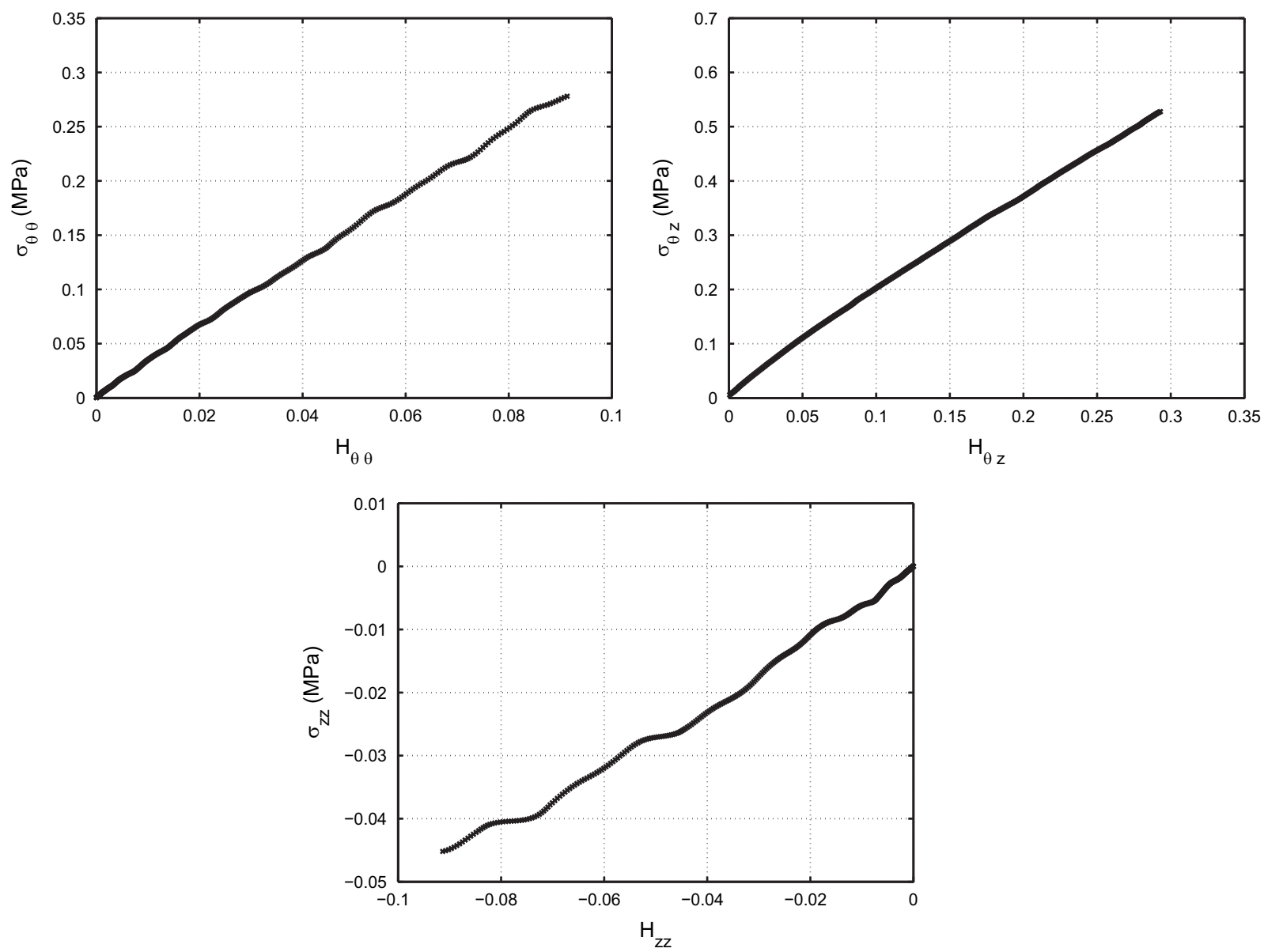

Fig. 4. Pure torsion: stress-strain curves.

$\lambda_{3}=\lambda$ and $\lambda_{1}=\lambda^{-1 / 2}$. Then, recalling Eq. (41),

$\bar{W}_{1}=\sigma_{\theta \theta}=0$ and $\bar{W}_{3}=\frac{\sigma_{z z}}{\lambda}$.

The initial values of $\bar{W}_{1}$ and $\bar{W}_{3}$ in Fig. $7(\mathrm{c})$ and (d) respectively correspond to those of $\sigma_{\theta \theta}$ and $\sigma_{z z}$ in Fig. 5. Then, during torsion, $\bar{W}_{1}$ and $\bar{W}_{3}$ evolve with $\tau$ similarly than $\sigma_{\theta \theta}$ and $\sigma_{z z}$ : first we observe a fast decrease due to the tensile stress relaxation, then they stabilize to a quite constant value explained by the negligible contribution of torsion on the stress as compared to the one of pre-stretching.

\subsubsection{With respect to the Hencky strain invariants $\left(K_{i}\right)_{i=2,3}$}

Finally, we study the derivatives of the strain energy with respect to the Hencky strain invariants. The results are presented in Fig. 8. The quantities are denoted $W_{K 2}=\partial W / \partial K_{2}$ and $W_{K 3}=\partial W / \partial K_{3}$.

Pure torsion: In the case of pure torsion, $K_{3}=0, W_{K 2}$ and $W_{K 3}$ are plotted with respect to $K_{2}$ as shown in Fig. 8(a) and (b). Unlike the derivatives with respect to $\left(I_{i}\right)_{i=1,2}$, there is no singularity especially at low angles, i.e. low $K_{2}$ : both $W_{K 2}$ and $W_{K 3}$ tend to zero as $K_{2}$ does. Finally, one can note that $W_{K 2}$ varies linearly with $K_{2}$.

Pre-stretching followed by torsion: Fig. 8(c) and (d) shows the evolution of the above-mentioned quantities with $\tau$ during torsion after pre-stretching $\lambda=1.75$ and 2.5. First, in Fig. 8(c), initial values of $W_{K 2}$ are those corresponding to the ones attained at the end of prestretching. During torsion, $W_{K 2}$ first decreases due to stress relaxation, then it increases as $\tau$ increases. It is to note that during torsion, $K_{3}$ changes from 1, which corresponds to uniaxial tension, to 0.993 .

Second, Fig. 8(d) exhibits a singularity: $W_{K 3}$ tends to infinity as $K_{3}$ tends to 1 . Nevertheless, we have checked that the term $W_{K 3} \boldsymbol{Y}$ tends to 0 as $K_{3}$ tends to 1 , then it has no influence on the measure of stress (see Eq. (49) and Remark 1). In order to explain the behavior of $W_{K 3}$ in the neighborhood of $\tau=0$, two assumptions can be invoked:

- either $W_{K_{3}}$ actually tends to infinity as $K_{3}$ tends to 1 , but slower than $\boldsymbol{Y}$ tends to 0,

- or the measurement error is amplified during the calculation of $W_{K_{3}}$ as argued by Criscione [6]. Thus, in this case the experimental determination of $W_{K 3}$ is irrelevant.

In order to decide between these two possibilities, we investigate the theoretical response of a neo-Hookean material $(C=0.35)$ subjected to a deformation process during which $K_{2}$ remains equal to 2 , and $K_{3}$ varies. The corresponding evolution of $W_{K 3}$ with respect to $K_{3}$ is presented in Fig. 9. This figure does not present any singularity: $W_{K 3}$ varies linearly with $K_{3}$. Even if this example does not constitute a rigorous proof, we are inclined to consider that the second above-mentioned assumption is the right one: considering experimental values of $W_{K 3}$ in the neighborhood of $K_{3}=1$ is not relevant. Thus, it corroborates the conclusion drawn by Criscione [6].

\section{Discussion}

Three methods are available to identify the hyperelastic constitutive equation for a given rubber-like material with tensiontorsion experimental results. In the following, they are presented from the most to the less restrictive, i.e. from the one that 

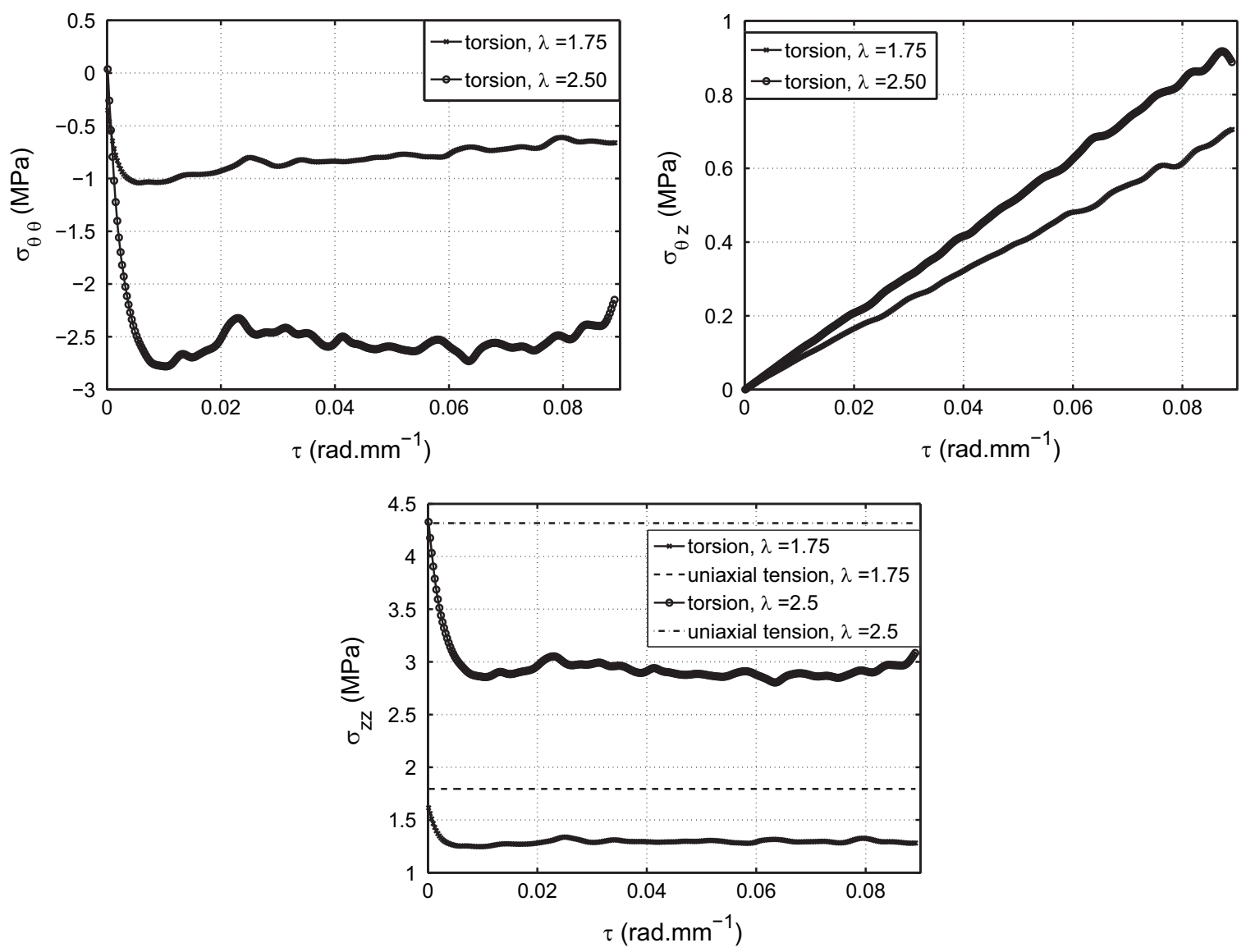

Fig. 5. Pre-stretching followed by torsion: Cauchy stress versus angle of twist per unit of length.

a

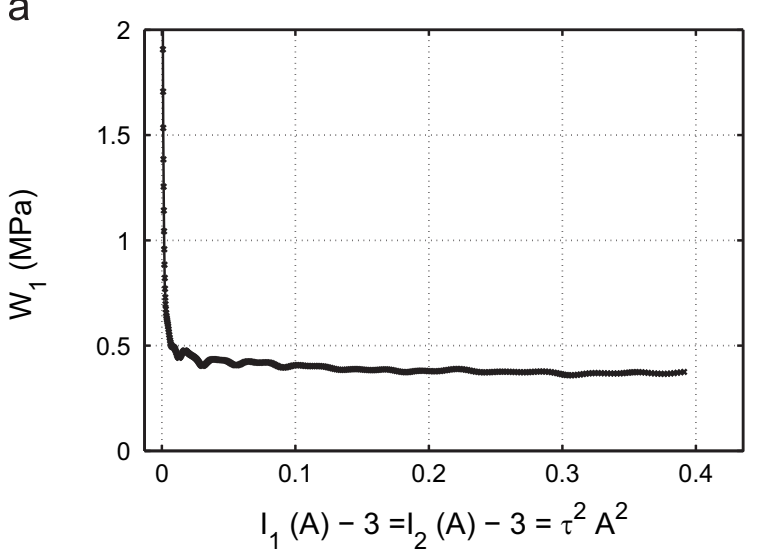

C

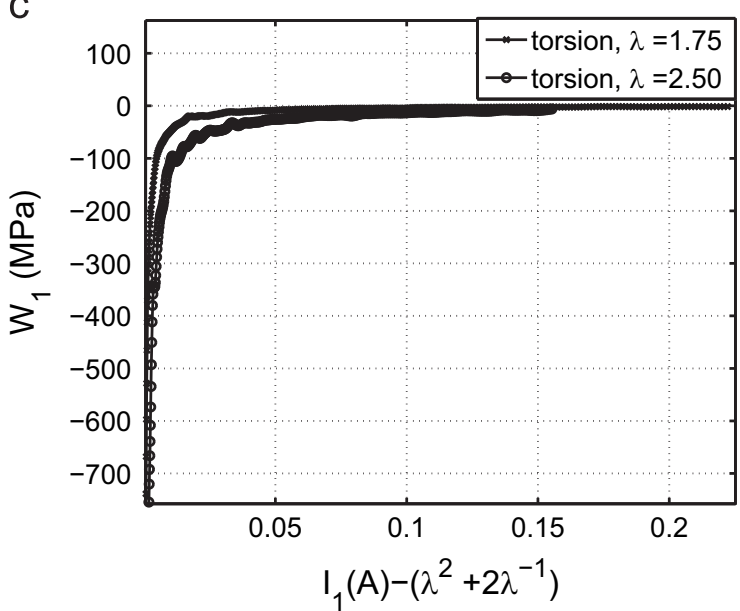

b

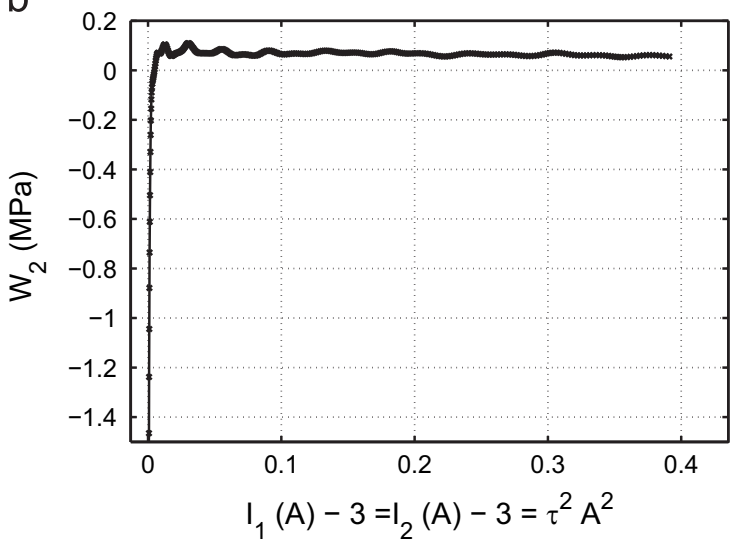

d

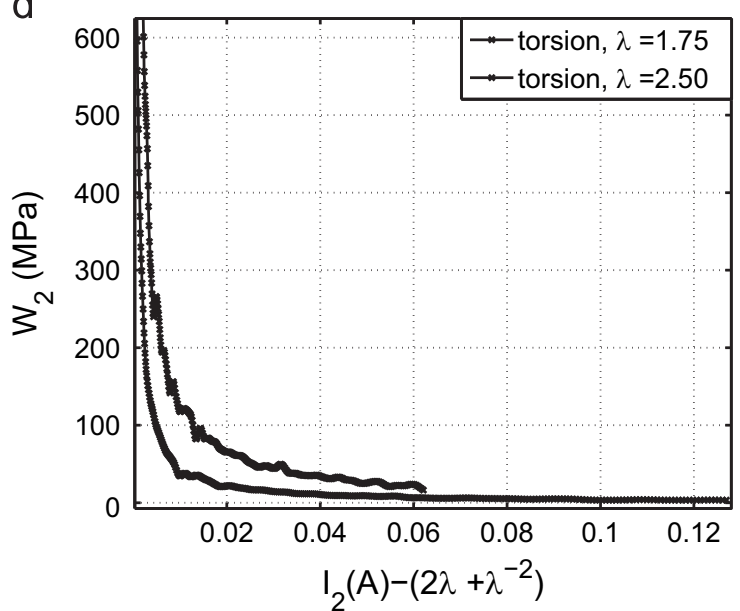

Fig. 6. Derivatives of the strain energy with respect to shifted $I_{1}$ and $I_{2}$. Top: pure torsion, bottom: pre-stretching followed by torsion. 
a

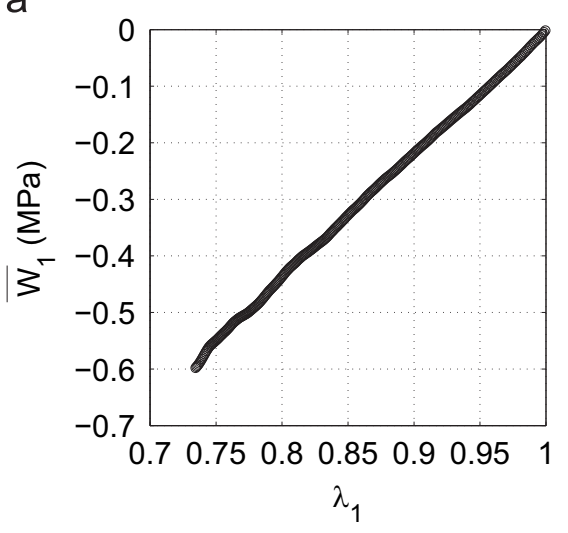

C

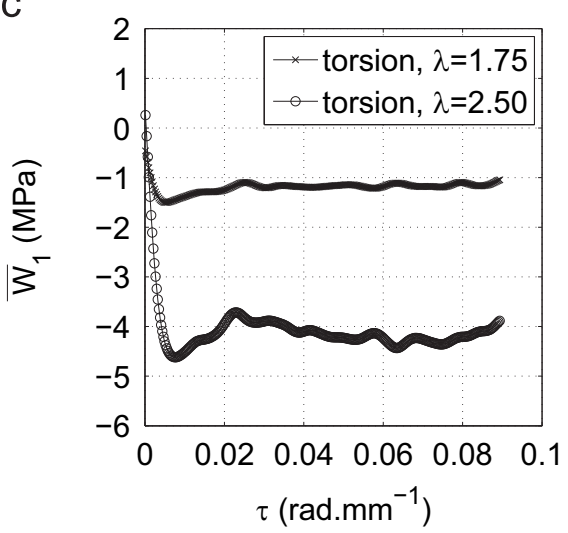

b

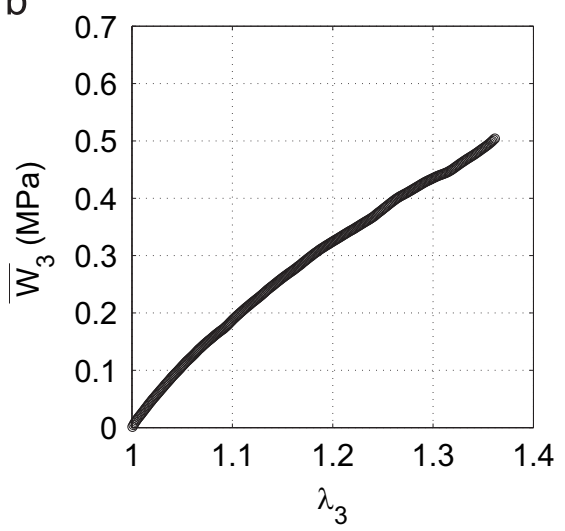

d

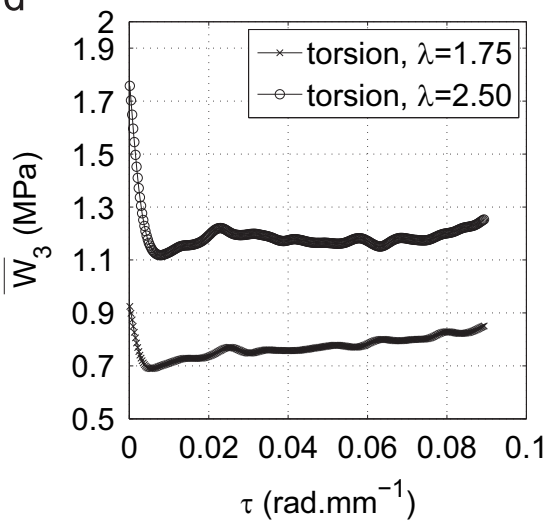

Fig. 7. Derivatives of the strain energy with respect to $\lambda_{1}$ and $\lambda_{3}$, and angle per unit of length. Top: pure torsion, bottom: pre-stretching followed by torsion.

necessitates the more assumptions to the one that necessitates the less.

Perform simultaneous tension-torsion experiments and focus on measured load and torque: In this case, it is not possible to directly calculate the derivatives of the strain energy function, and then the stresses. However, one can postulate a priori the strain energy function. Then, by replacing $W_{1}$ and $W_{2}$ by their explicit forms in terms of the material parameters and the strain in load and torque expressions given by Eqs. (25)-(26), the material parameters can be determined by a classical fitting method. From an experimental point of view, this method is obvious and has already been considered by several authors (see for example $[27,11]$ ). However, from a theoretical point of view it is questionable because the early choice of the strain energy function renders it very restrictive. Indeed, it is well-known that the choice of a hyperelastic constitutive equation able to predict the response of rubberlike material under multiaxial loading conditions is a complex problem [20].

Perform pre-stretching followed by torsion experiments and focus on the derivatives of the strain energy function: When the uniaxial extension $\lambda$ is kept constant during torsion, the calculation of the derivatives of the strain energy function from experimental measurements of $N$ and $M$ at the outer radius of the cylinder becomes possible. However, it has been exhibited that the experimental determination of the derivatives of the strain energy function with respect to the classical Cauchy-Green strain invariants $I_{1}$ and $I_{2}$ is irrelevant at low strains as shown for example in Fig. 6. Many other authors have encountered the same difficulties with tension-torsion tests [26,22] and biaxial tests $[1,23,34,14$, $15,8,17,5]$. In most of these cases, authors have excluded experimental values at low strain arguing that it is the consequence of measurement errors. In fact, this difficulty is inherent to the mutual dependency between the terms that appear when the derivative of the strain energy function with respect to $\boldsymbol{B}$ is decomposed into the derivatives of the strain energy function with respect to $I_{1}$ and $I_{2}$. This problem has been thoroughly investigated by Criscione [6] for biaxial loading conditions. Criscione demonstrated that for incompressible hyperelastic materials the calculation errors in the derivatives of the strain energy function are magnified by the factor $k$ given by

$k=\frac{1}{\sqrt{1-R_{C}\left(\operatorname{dev} \boldsymbol{B}, \operatorname{dev} \boldsymbol{B}^{-1}\right)^{2}}}$,

where the function $R_{c}(\boldsymbol{P}, \boldsymbol{Q})$ quantifies the covariance amongst tensors $\boldsymbol{P}$ and $\boldsymbol{Q}$ as

$R_{C}(\boldsymbol{P}, \boldsymbol{Q})=\frac{\operatorname{abs}(\boldsymbol{P}: \mathbf{Q})}{\sqrt{\boldsymbol{P}: \boldsymbol{P}} \sqrt{\mathbf{Q}: \mathbf{Q}}}$

Practically, $R_{C}$ takes values in $[0,1]$ and the higher the covariance the higher the mutual dependency. So, if $R_{C}\left(\operatorname{dev} \boldsymbol{B}, \operatorname{dev} \boldsymbol{B}^{-1}\right)$ tends to 1 then $k$ tends to infinity and consequently the calculation errors in the derivatives of $W$ are very high. We now apply this approach to the special case of tension-torsion: Fig. 10 presents the values of $R_{C}$ as a function of the loading conditions $\lambda$ and $\tau$. Clearly, for most of the pairs $(\lambda, \tau) R_{C}$ is very close to 1 , especially for low values of the angle of twist. Thus, for tension-torsion experiments, the use of the Cauchy-Green invariants $I_{1}$ and $I_{2}$ leads to large calculation errors.

In contrast with Cauchy-Green invariants, for both the principal stretch ratios $\lambda_{1}$ and $\lambda_{3}$, and the Hencky strain invariants $K_{2}$ and $K_{3}$ the terms involved in the expression of the Cauchy stress tensor are mutually orthogonal, i.e. $R_{C}=0$. This property guarantees the independence of the corresponding derivatives of the 
a

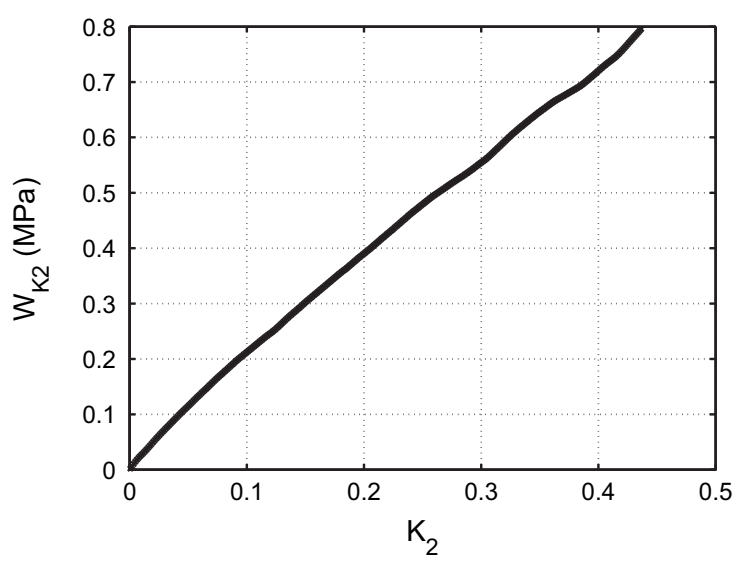

C

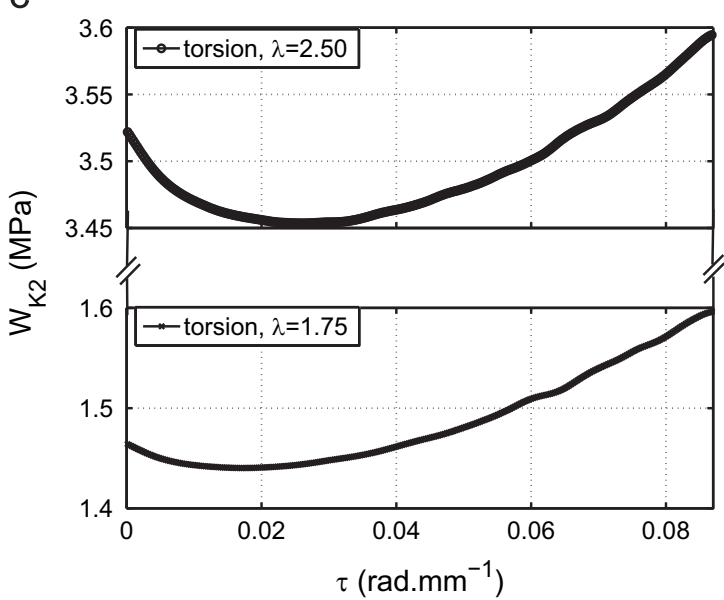

b

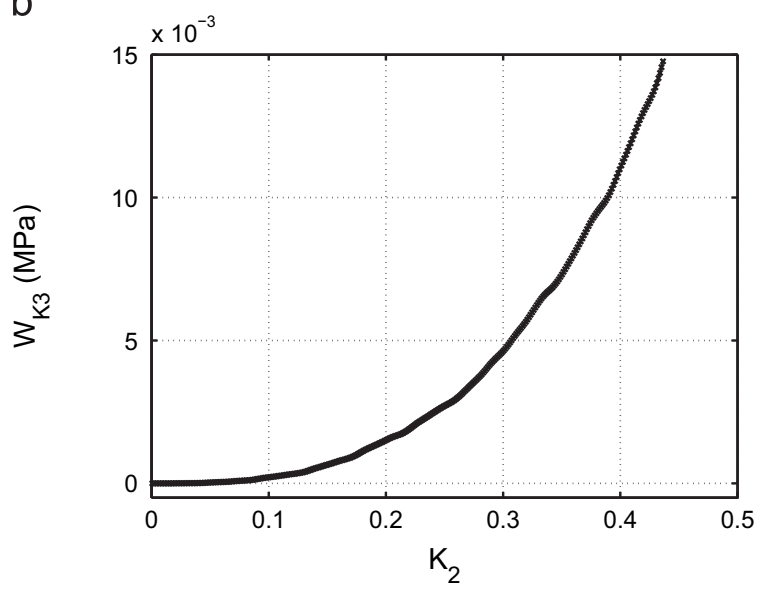

d

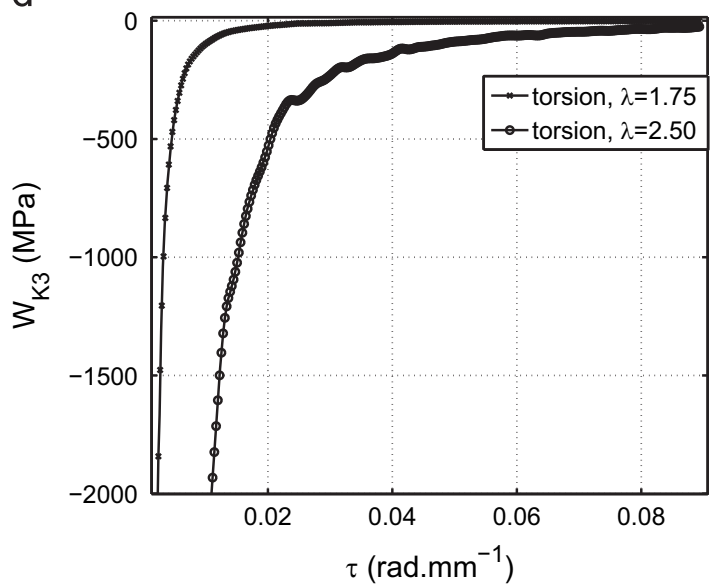

Fig. 8. Derivatives of the strain energy with respect to $K_{2}$ and $K_{3}$, and angle per unit of length. Top: pure torsion, bottom: pre-stretching followed by torsion.

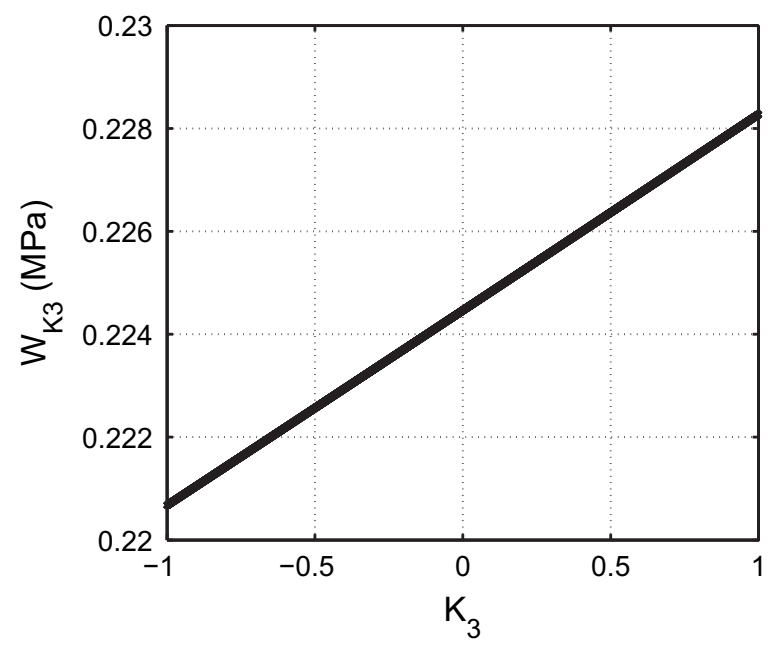

Fig. 9. Deformation of a neo-Hookean material: change in $W_{K 3}$ with $K_{3}$, as $K_{2}$ remains constant $(=2)$.

strain energy functions. To give a simple illustration of this analysis, consider the case of uniaxial tension for which the only non-zero Cauchy stress is $\sigma_{z z}$. Recalling Eqs. (9), (41) $)_{2}$ and (49), $\sigma_{z z}$ is given by

$\sigma_{z z}=2\left(\lambda W_{1}+W_{2}\right)\left(\lambda-\frac{1}{\lambda^{2}}\right)=\sqrt{\frac{3}{2}} W_{K 2}=\lambda_{3} \bar{W}_{3}$.

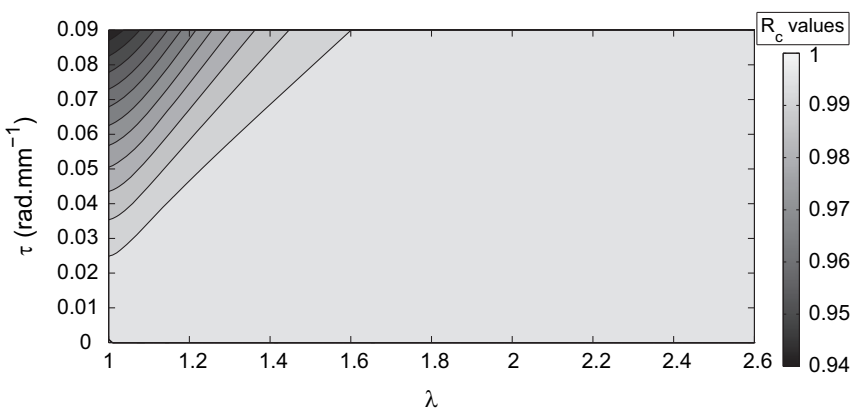

Fig. 10. Values of covariance for several cases of tension-torsion.

Consider that $\sigma_{z z}$ has been obtained from experimental data, then Eq. (58) ${ }_{1}$ shows that $W_{1}$ and $W_{2}$ remain undetermined, Eq. (58) immediately leads to $W_{K 2}$ while $W_{K 3}$ is undetermined, and Eq. $(58)_{3}$ immediately gives $\bar{W}_{3}$ while $\bar{W}_{1}=0$ is deduced from $\sigma_{\theta \theta}=0$.

To conclude, this method requires some experimental and theoretical restrictions, but it is the most appropriate to collect relevant information to choose the strain energy function. Nevertheless, the choice of the set of invariants to consider is of importance to apply the method:

- $\left(I_{i}\right)_{i=1,2}$ induce a covariance equal to 1 for stress terms and then lead to calculation errors for small torsional strain, 
- $\left(K_{i}\right)_{i=2,3}$ lead to orthogonal stress terms and then permit a satisfactory determination of $W_{K 2}$, but the determination of $W_{K 3}$ is flawed as $K_{3}$ is close to 1, i.e. for uniaxial tension,

- $\lambda_{i=1,3}$ is the most relevant set of invariants to determine the derivatives of the strain energy function.

Perform pre-stretching followed by torsion experiments and focus on stresses: Experiments are the same as the previous method: the uniaxial extension must remain constant during torsion. Whatever the set of invariants chosen, the Cauchy stress tensor can be determined at the outer radius of the cylinder. It is calculated from strain energy derivatives with respect to $I_{1}$ and $I_{2}$. The combination of both strain energy derivatives allows us to bypass abovementioned problems at small strain. This simple calculation extends the work of Penn and Kearsley [26] to the determination of the Cauchy stress tensor.

\section{Conclusion}

It is well-recognized that the best method to determine hyperelastic constitutive equation of a given rubber-like material consists in calculating the derivatives of the strain energy. In this paper, it has been established that the strain energy derivatives can be easily calculated using experimental data issued from pre-stretching followed by torsion tests if a relevant set of mechanical quantities (strain invariants, stretch ratios) is adopted. This result is particularly useful to compare the efficiency of hyperelastic constitutive equations, i.e. strain energy functions, for a given rubber-like material, and to determine the most appropriate one to predict its response under quasi-static multiaxial loading conditions.

The starting point of the study was the application of the Penn and Kearsley [26] method to calculate the derivatives of the strain energy with respect to the Cauchy-Green strain invariants $\left(\left(I_{i}\right)_{i=1,2}\right)$ to pure torsion and pre-extension followed by torsion experimental results. It has been shown that this set of invariants does not permit to determine the derivatives of the strain energy because they are highly correlated. Considering a similar method, we have demonstrated that two other sets of mechanical quantities, i.e. the principal stretch ratios $\left(\lambda_{i}\right)_{i=1,3}$ and the Hencky strain invariants $\left(\left(K_{i}\right)_{i=2,3}\right)$ introduced by Criscione [6], are theoretically well-suited for the derivation of strain energy derivatives because they are associated to mutually orthogonal terms in Cauchy stress tensor components. Nevertheless, as measurement errors are highly amplified for the strain energy derivatives with respect to $K_{3}$ when $K_{3}^{2}$ tends to 1 , i.e. for uniaxial tension, the best set of mechanical quantities to retain is the one of principal stretch ratios.

Finally, we have established that Cauchy stress components can be directly calculated from force and torque measurements. In fact this calculation is independent from the discussion on wellconditioned representation of strain states and leads to the same results for each set of invariants. This result is noticeable because it applies to heterogeneous deformation and does not require to presuppose the form of the constitutive equation. Moreover, it pursues the results of the pioneering work of Penn and Kearsley [26]. It is worth noting that this method can be used for compressible materials [21] and could also be applied to hyperviscoelastic constitutive equations based on summation of strain energy functions.

\section{Acknowledgments}

The authors are grateful to Renault S.A.S. for financing this project and particularly Dr. A.-S. Béranger and Mr. F. Le Brazidec for their supervision and helpful discussions.

\section{References}

[1] G.W. Becker, On the phenomenological description of the nonlinear deformation of rubberlike high polymers, J. Polym. Sci. C16 (1967) 2893-2903.

[2] E.W. Billington, The Poynting effect, Acta Mech. 58 (1986) 19-31.

[3] P.J. Blatz, W.L. Ko, Application of finite elastic theory to the deformation of rubbery materials, Trans. Soc. Rheol. 6 (1962) 223-251.

[4] M.C. Boyce, E.M. Arruda, Constitutive models of rubber elasticity: a review, Rubber Chem. Technol. 73 (2000) 504-523.

[5] L. Chevalier, Y. Marco, Tools for multiaxial validation of behavior laws chosen for modeling hyper-elasticity of rubber-like materials, Polym. Eng. Sci. 42 (2002) 280-298.

[6] J.C. Criscione, Rivlin's representation formula is ill-conceived for the determination of response functions via biaxial testing, J. Elast. 70 (2003) 129-147.

[7] J.C. Criscione, J.D. Humphrey, A.S. Douglas, W.C. Hunter, An invariant basis for natural strain which yields orthogonal stress response terms in isotropic hyperelasticity, J. Mech. Phys. Solids 48 (2000) 2445-2465.

[8] Y. Fukahori, W. Seki, Molecular behaviour of elastomeric materials under large deformation: 1. Re-evaluation of the Mooney-Rivlin plot, Polymer 33 (1991) 502-508.

[9] D. Haines, W. Wilson, Strain-energy density function for rubberlike materials, J. Mech. Phys. Solids 27 (1979) 345-360.

[10] W.H. Han, F. Horka, G.B. McKenna, Mechanical and swelling behaviors of rubber: a comparison of some molecular models with experiment, Math Mech. Solids 4 (1999) 139-167.

[11] S. Hartmann, Numerical studies on the identification of the material parameters of Rivlin's hyperelasticity using tension-torsion tests, Acta Mech. 148 (2001) 129-155.

[12] G.A. Holzapfel, Nonlinear Solid Mechanics. A Continuum Approach for Engineering, J. Wiley and Sons, Chichester, 2000.

[13] J.D. Humphrey, R.L. Barazotto Jr., W.C. Hunter, Finite extension and torsion of papillary muscles: a theoretical framework, J. Biomech. 25 (1992) 541-547.

[14] D.F. Jones, L.R.G. Treloar, The properties of rubber in pure homogeneous strain, J. Phys. D: Appl. Phys. 8 (1975) 1285-1304.

[15] S. Kawabata, H. Kawai, Strain energy density functions of rubber vulcanizates from biaxial extension, Adv. Polym. Sci. 24 (1977) 89-124.

[16] S. Kawabata, M. Matsuda, K. Tei, H. Kawai, Experimental survey of the strain energy density function of isoprene rubber vulcanizate, Macromolecules 14 (1981) 154-162.

[17] J. Lambert-Diani, C. Rey, New phenomenological behavior laws for rubbers and thermoplastic elastomers, Eur. J. Mech. A/Solids 18 (1999) 1027-1043.

[18] A.S. Lectez, E. Verron, B. Huneau, A.S. Beranger, F. Le Brazidec, Characterization of elastomers under simultaneous tension and torsion for application to engine mounts, in: Proceedings of the 8th European Conference on Constitutive Models for Rubber (ECCMR), vol. 8, 2013, pp. 585-590.

[19] G. Machado, D. Favier, G. Chagnon, Membrane curvatures and stress-strain ful fields of axisymmetric bulge tests from 3D-DIC measurements. Theory and validation on virtual and experimental results, Exp. Mech. 52 (2012) 865-880.

[20] G. Marckmann, E. Verron, Comparison of hyperelastic models for rubberlike materials, Rubber Chem. Technol. 79 (2006) 835-858.

[21] G.B. McKenna, L.J. Zapas, The time dependent strain potential function for a polymeric glass, Polymer 26 (1985) 543-550.

[22] G.B. McKenna, L.J. Zapas, On the small strain behavior of peroxide crosslinked natural rubber, Rubber Chem. Technol. 59 (1986) 130-137.

[23] Y. Obata, S. Kawabata, H. Kawai, Mechanical properties of natural rubber vulcanizates in finite deformation, J. Polym. Sci. Part A-2: Polym. Phys. 8 (1970) 903-919.

[24] R.W. Ogden, Large deformation isotropic elasticity - on the correlation of theory and experiment for incompressible rubberlike solids, Proc. R. Soc. Lond. A. Math. Phys. Sci. 326 (1972) 565-584.

[25] R.W. Ogden, G. Saccomandi, I. Sgura, Fitting hyperelastic models to experimental data, Comput. Mech. 34 (2004) 484-502.

[26] R.W. Penn, E.A. Kearsley, The scaling law for finite torsion of elastic cylinders, Trans. Soc. Rheol. 20 (1976) 227-238.

[27] R.S. Rivlin, D.W. Saunders, Large elastic deformations of isotropic materials VII. Experiments on the deformation of rubber, Philos. Trans. R. Soc. Lond. Ser. A Math. Phys. Sci. 243 (1951) 251-288.

[28] M. Sasso, G. Palmieri, G. Chiappini, D. Amodio, Characterization of hyperelastic rubber-like materials by biaxial and uniaxial stretching tests based on optical methods, Polym. Test. 27 (2008) 995-1004.

[29] T. Sendova, J.R. Walton, On strong ellipticity for isotropic hyperelastic materials based upon logarithmic strain, Int. J. Non-Linear Mech. 40 (2005) 195-212.

[30] P. Steinmann, M. Hossain, G. Possart, Hyperelastic models for rubber-like materials: consistent tangent operators and suitability for Treloar's data, Arch. Appl. Mech. 82 (2012) 1183-1217.

[31] L.R.G. Treloar, Strains in an inflated rubber sheet, and the mechanism of bursting, Trans. Inst. Rubber Ind. 19 (1944) 201-212.

[32] L.R.G. Treloar, Stress-strain data for vulcanised rubber under various types of deformation, Trans. Faraday Soc. 40 (1944) 59-70.

[33] L.R.G. Treloar, Stresses and birefringence in rubber subjected to general homogeneous strain, Proc. Phys. Soc. 60 (1948) 135.

[34] L.R.G. Treloar, The Physics of Rubber Elasticity, Oxford University Press Oxford, 1975.

[35] T. Tsakalakos, The bulge test: a comparison of the theory and experiment for isotropic and anisotropic films, Thin Solid Films 75 (1981) 293-305. 